\title{
Prevalence and Risk Factors of Hyperuricemia in Guangming District, Shenzhen: An Observational Study from a District Hospital in 2020
}

\author{
CHEN Kaiyin ${ }^{1}$; XIAO Ping ${ }^{1}$; WANG Tingwei ${ }^{1}$; LI Shaofang ${ }^{1}$
}

Health Management Center, the Seventh Affiliated Hospital of Sun Yat-sen University, Shenzhen, Guangdong Province, China, 518107

E-mail:523279326@qq.com https://orcid.org/0000-0002-6568-0268

Article History Received 26 June 2021 Accepted 25 July 2021 Published 30 September 2021 Cite this Article CHEN Kaiyin; XIAO Ping; WANG Tingwei; LI Shaofang.Prevalence and Risk Factors of Hyperuricemia in Guangming District, Shenzhen: An Observational Study from a District Hospital in 2020 [J].Medical Research, 2021.3(3):33-38, http://dx.doi.org/mrhk/10.6913/MRHK.202109_3(3).0004

Copyright $@ 2021$ Creative Publishing Co., Limited.All rights reserved.Email:mrhk@mrhk.cc

\section{ABSTRACT}

Objective This study aimed to explore the prevalence of hyperuricemia among the general adult population in Shenzhen and to determine the risk factors of hyperuricemia.

Methods A total of 9337 cases who underwent physical examination in The Seventh Affiliated Hospital of Sun Yat-Sen University in 2020 were enrolled in this study. The binary logistic regression analysis was performed to determine potential risk factors of hyperuricemia.

Result The overall prevalence rate of hyperuricemia in the studied population was $40.5 \%$ (3783/9337). Subgroup analysis showed that the prevalence of males was 52.8\% (3046/5769) and the prevalence of females was $20.7 \%$ (737/3568). Moreover, among different age groups ( $\leq 29$ years old, 30-39 years old, 40-49 years old, 50-59 years old, and $\geq 60$ years old), the prevalence rate of males was significantly higher than that of females $(\mathrm{P}<0.05)$. To further determine the relationship between the body mass index (BMI) and hyperuricemia, the population with normal BMI was set as the control group. After controlling for gender and age, the prevalence rate of hyperuricemia in the overweight population increased by 1.799 times; and the prevalence rate in the obese and very obese population increased by 3.283 times and 3.830 times, respectively.

Conclusion The data presented in this study demonstrated that the prevalence rate of hyperuricemia is high in Shenzhen, especially in males. Moreover, the level of BMI is significantly linked to the prevalence of hyperuricemia. Therefore, early interventions should be carried out to address the high prevalence of hyperuricemia in male and obese populations.

Keywords: Physical Examination; hyperuricemia; Body mass index; Shenzhen

\section{INTRODUCTION}

Hyperuricemia refers to the abnormal increase in the level of uric acid in the blood. It has been associated with high risks of gout, diabetes, and cardiovascular diseases ${ }^{[1,2]}$. With the 
improvement of the economy and the change in lifestyle and dietary patterns, the incidences of hyperuricemia and obesity are both on the rise, posing a significant threat to public health. Particularly, in recent years, the incidence of hyperuricemia in China has shown a notable increase at younger ages ${ }^{[3]}$. It is reported that the current overall prevalence of hyperuricemia in China is $13.3 \%$, making it the fourth "Hyper" after the triple $\mathrm{H}$ (hyperglycemia, hypertension, and hyperlipidemia) ${ }^{[4]}$. Therefore, more attention should be paid to better understand the situation of hyperuricemia and seek strategies for prevention.

Previous studies have shown that the prevalence of hyperuricemia varied among different regions. Shenzhen, as one of the largest cities in China, is a rapidly developing city with a population of 12.59 million; and it may have a higher prevalence rate of hyperuricemia than the national average. The aim of this study was twofold: a) to explore the latest prevalence of hyperuricemia in populations of different ages and genders in Guangming District, Shenzhen; b) to determine the relationship between hyperuricemia and body mass index (BMI). We hope that the results of this study will help improve medical staff's awareness of high-risk populations of hyperuricemia and help target populations receive corresponding health guidance.

\section{METHODS}

\section{Research participants}

Citizens who had a physical examination at the Health Management Center of the Seventh Affiliated Hospital of Sun Yat-sen University in the year 2020 were enrolled as research participants. The inclusion criteria were: 1) over 18 years old; 2) no missing information on blood uric acid, BMI, blood pressure, fasting blood glucose levels, blood lipid levels, or other important medical histories. Eventually, a total of 9337 subjects (5769 males and 3568 females) were included in the study, with an average age of $36.81 \pm 10.28$ years old.

\section{Blood test}

All the examinees had fasting venous blood sampling in the morning. Blood pressure, height, and body weight were also measured. The body mass index (BMI) was automatically generated by the height and weight integrated machine. The Abbott ARCHITECT c16000 instrument was used to measure serum uric acid, fasting blood glucose, total cholesterol, triglycerides, low-density lipoprotein cholesterol, and high-density lipoprotein cholesterol. Before the test, the parameters were set in strict accordance with the instructions, and the calibration and quality control were performed. The quality control determination was performed before and after the measurement to ensure the reliability of the test results.

\section{Diagnostic criteria}

According to the diagnostic criteria of the seventh edition of "Internal Medicine" (textbook), men and postmenopausal women with blood uric acid greater than $420 \mu \mathrm{mol} / \mathrm{L}$, and premenopausal women with blood uric acid greater than $350 \mu \mathrm{mol} / \mathrm{L}$ were diagnosed as hyperuricemia. According to the Chinese weight classification standards, BMI below $18.5 \mathrm{~kg} / \mathrm{m}^{2}$ is considered thin, BMI between $18.5-23.9 \mathrm{~kg} / \mathrm{m}^{2}$ is considered normal, BMI between $24-27.9 \mathrm{~kg} / \mathrm{m}^{2}$ is considered overweight, and BMI between $28 \sim 31.9 \mathrm{~kg} / \mathrm{m}^{2}$ is 
considered obese, and BMI greater than $32 \mathrm{~kg} / \mathrm{m}^{2}$ is considered very obese.

\section{Statistical analysis}

The Excel (Microsoft) software was used for data import and the SPSS software was used for statistical analysis. The statistical description of count data was expressed by frequency and percentage. The Chi-square test was used to analyze the prevalence of hyperuricemia among populations with different BMI levels. The binary Logistic regression analysis was used to determine the impact of BMI on hyperuricemia. A two-sided test was used in the study and $\mathrm{P}<0.05$ indicated that the difference was statistically significant.

\section{RESULTS}

\section{Prevalence rates of hyperuricemia among the populations of different age and gender}

As shown in Table 1, a total of 9337 individuals participated in this study. Their average age was $36.81 \pm 10.28$ years old. In the physical examination, 3783 participants $(40.5 \%)$ were diagnosed with hyperuricemia. The overall prevalence rate of men with hyperuricemia was $52.8 \%$ (3046/5769), and the overall prevalence rate of women was $20.7 \%$ (737/3568). As shown in Table 1, the overall prevalence rate of hyperuricemia differs among different age groups ( $\leq 29$ years old, $30-39$ years old, $40-49$ years old, 50-59 years old, and $\geq 60$ years old). Moreover, in each age group, the hyperuricemia prevalence rate of men was significantly higher than that of women (all $\mathrm{p}$ values $<0.05$ ).

\begin{tabular}{|c|c|c|c|c|c|c|c|c|c|}
\hline \multirow{3}{*}{$\begin{array}{c}\text { Age } \\
\text { (Years) }\end{array}$} & \multicolumn{3}{|c|}{ Overall } & \multicolumn{3}{|c|}{ Male } & \multicolumn{3}{|c|}{ Female } \\
\hline & No. with & & & No. with & & & No. with & & \\
\hline & hyperuricemia & Total & Rate & hyperuricemia & Total & Rate & hyperuricemia & Total & Rate \\
\hline$\leq 29$ & 1134 & 2713 & $41.80 \%$ & 874 & 1571 & $55.63 \%$ & 260 & 1142 & $22.77 \%$ \\
\hline $30-39$ & 1396 & 3323 & $42.01 \%$ & 1142 & 2070 & $55.17 \%$ & 254 & 1253 & $20.27 \%$ \\
\hline $40-49$ & 834 & 2135 & $39.06 \%$ & 670 & 1366 & $49.05 \%$ & 164 & 769 & $21.33 \%$ \\
\hline $50-59$ & 348 & 899 & $38.71 \%$ & 313 & 631 & $49.60 \%$ & 35 & 268 & $13.06 \%$ \\
\hline$\geq 60$ & 71 & 269 & $26.39 \%$ & 47 & 131 & $35.88 \%$ & 24 & 138 & $17.39 \%$ \\
\hline Total & 3783 & 9339 & $40.5 \%$ & 3046 & 5769 & $52.8 \%$ & 737 & 3570 & $20.6 \%$ \\
\hline
\end{tabular}

years old, and $\geqslant 60$ years old). Moreover, in each age group, the hyperuricemia prevalence rate of men was significantly higher than that of women (all $p$ values $<0.05$ ).

Table 1. The prevalence of hyperuricemia in different gender and age groups.

\section{Risk factors for hyperuricemia}

The presence or absence of hyperuricemia was used as the dependent variable, and gender, age, and BMI were the independent variables. The binary logistic regression analysis was performed. As shown in Table 2, male, middle-aged, and high BMI levels (overweight, obese, or very obese) were risk factors for hyperuricemia $(\mathrm{P}<0.005)$. Next, the group with normal BMI level was set as the standard control. After controlling for gender and age, the prevalence rate of hyperuricemia in the overweight group increased by 1.799 times, and that of the obese and very obese groups increased by 3.283 times and 3.830 times, respectively. In contrast, the 
prevalence rate of hyperuricemia in the thin group was only 0.477 times that of the normal group.

\begin{tabular}{lcccc}
\hline \multicolumn{2}{c}{ Factors } & P value & OR & $95 \% \mathrm{Cl}$ \\
\hline Gender & Male & & & \\
& Female & $<0.005$ & 0.349 & $0.314 \sim 0.388$ \\
Age (Years) & $\leq 29$ & & \\
& $30-39$ & & 2.895 & $2.113 \sim 3.967$ \\
& $40-49$ & & 2.049 & $1.502 \sim 2.795$ \\
& $50-59$ & 1.499 & $1.098 \sim 2.047$ \\
BMI & $\geq 0.005$ & 1.311 & $0.946 \sim 1.817$ \\
& Normal & & & \\
& Thin & & 0.477 & $0.358 \sim 0.634$ \\
& Overweight & $<0.005$ & 1.799 & $1.616 \sim 2.001$ \\
& Obese & & 3.283 & $2.793 \sim 3.858$ \\
& Very obese & & 3.83 & $2.766 \sim 5.305$ \\
\hline
\end{tabular}

Table 2. Risk factors for hyperuricemia

\section{DISCUSSION}

In this study, the prevalence rate of hyperuricemia among the adult population in Shenzhen was $40.5 \%$, which was much higher than the overall national level. Regional difference on the prevalence rate of hyperuricemia has been reported. A meta-analysis on the prevalence of hyperuricemia in mainland China from 2000 to $2019^{[5]}$ showed that the prevalence of hyperuricemia in the southern, southwestern, and northeastern regions were $25.5 \%, 21.2 \%$ and 15.9, respectively. In particular, the prevalence rate in Guangdong has been reported to be as high as $42.2 \%$, which is comparable to the rate determined in this study.

Multiple reasons may contribute to the high prevalence rate of hyperuricemia in Shenzhen. First, as a first-tier city in China, Shenzhen is dominated by young generations. Compared with the population in underdeveloped rural areas, people in developed cities with higher living standards consume more food and drinks posing higher risks of hyperuricemia, such as purine-rich animal products, high-fat foods, and alcohol ${ }^{[6]}$. Moreover, Guangming District in Shenzhen is an ecological high-tech industrial new city. The citizens have high work pressure and stress, which is also associated with their unhealthy lifestyle. Therefore, it is reasonable to speculate that these factors derived from the city and the population may contribute to the high prevalence rate of hyperuricemia.

The results of this study highlighted the gender difference of hyperuricemia in the physical examination population of Guangming District, Shenzhen. The prevalence rate of male hyperuricemia decreased with age while the prevalence rate of female hyperuricemia 
increased with age. The study by Lin Xiyang et al. ${ }^{[7]}$ reported that young men and women aged $\geq 50$ years should actively pay attention to blood uric acid levels, and that the trend of men's uric acid levels with age is associated with glucose metabolism. The prevalence rate of female hyperuricemia is 0.349 times that of men, and the risk of hyperuricemia in women is lower than that in men. This phenomenon may be related to the physiological differences between males and females. It is reported that androgens inhibit the excretion of uric acid in the body, while estrogen can make the organelles phospholipase susceptible to urate crystals and cause cellular reactions, resist crystal precipitation, and promote the excretion of uric acid [8].

Further, this study determined that BMI levels were positively correlated with the prevalence rate of hyperuricemia. Setting the normal BMI group as the baseline, the prevalence rate of hyperuricemia in the overweight, obese and very obese groups was progressively increasing. The prevalence rate of hyperuricemia in the physical examination population of the overweight group, obese group and very obese group was 1.799, 3.283, and 3.830 times that of the normal group, respectively. The prevalence rate of hyperuricemia in the physical examination population of the thin group was 0.477 times that of the normal group. These results indicate that BMI levels closely link to the hyperuricemia.

It is known that obesity is a risk factor for cardiovascular diseases and metabolic diseases, such as diabetes. Hyperuricemia is closely related to the components of the metabolic syndrome, and can even be classified as one of the components of the metabolic syndrome. Uric acid is a product of purine metabolism. Long-term unhealthy dietary habits, changes in dietary composition (high-purine foods) and long-term intake of alcohol can all lead to hyperuricemia and obesity. Obesity and hyperuricemia can mutually promote each other through various metabolic mechanisms [9]. Obese people accumulate visceral fat, and the acidic metabolites of lipolysis can inhibit uric acid excretion and indirectly promote the increase of blood uric acid ${ }^{[10]}$. In addition, obese patients often have insulin resistance, which is the main cause of decreased renal uric acid excretion. Hyperuricemia and insulin resistance accompanied by hyperinsulinemia increase uric acid reabsorption and decrease excretion ${ }^{[11]}$.

\section{SUMMARY}

The data presented in this study demonstrate the high prevalence rate of hyperuricemia in the general population in Guangming District, Shenzhen. The prevalence rate of hyperuricemia decreases with age in males, but it is increasing in females. Moreover, the data also marks the positive correlation between BMI levels and the prevalence rate of hyperuricemia. Therefore, in health management, corresponding education and health interventions need to be adopted for the different populations. In particular, for young, male, and obese populations who are at high risk of hyperuricemia, early intervention should be carried out to reduce the occurrence of adverse consequences.

\section{ACKNOWLEDGMENT}

None.

\section{STATEMENT}


There is no conflict of interest in this article.

\section{REFERENCES}

1 Dehghan A, van Hoek M, Sijbrands EJ, Hofman A, Witteman JC. High serum uric acid as a novel risk factor for type 2 diabetes. Diabetes Care. 2008 Feb; 31(2):361-2. doi: 10.2337/dc07-1276. Epub 2007 Oct 31.

2 Ni Q, Lu X, Chen C, Du H, Zhang R. Risk factors for the development of hyperuricemia: A STROBE-compliant cross-sectional and longitudinal study. Medicine (Baltimore). 2019 Oct; 98(42):e17597. doi: 10.1097/MD.0000000000017597.

3 Kuo CF, Grainge MJ, Zhang W, et al. Global epidemiology of gout: prevalence, incidence and risk factors[J]. Nat Rev Rheumatol, 2015, 11(11):649-662.

4 Liu R, Han C, Wu D, et al. Prevalence of hyperuricemia and gout in mainland China from 2000 to2014:a systematic review and meta-analysis[J].Biomed Res Int, 2015. 2015:762820.

5 Li Y, Shen Z, Zhu B, Zhang H, Zhang X, Ding X. Demographic, regional and temporal trends of hyperuricemia epidemics in mainland China from 2000 to 2019: a systematic review and meta-analysis. Glob Health Action. 2021 Jan 1; 14(1):1874652. doi:

10.1080/16549716.2021.1874652.

6 Miao Z, Li C, Chen Y, Zhao S, Wang Y, Wang Z, Chen X, Xu F, Wang F, Sun R, Hu J, Song W, Yan S, Wang CY. Dietary and lifestyle changes associated with high prevalence of hyperuricemia and gout in the Shandong coastal cities of Eastern China. J Rheumatol. 2008 Sep; 35(9):1859-64. Epub 2008 Jul 15.

7 LIN Xi-yang, YUAN Xue, LIU Jun-tian, CHEN Ying, et al.The uric acid level difference between males and females and the influencing factor of hyperuricemia in 45229 residents with the physical examination of Tianjin area [J].Chinese Journal of Prevention and Control of Chronic Diseases, 2016.24 (12): 904-908.

doi:10.16386/j.cjpccd.issn.1004-6194.2016.12.006.

8 Xuelian Yong, Yong Ye. Analysis of the blood uric acid test results of a college freshman's health examination [J]. Chinese Journal of School Health， 2014， 35 (9): 1430- 1431. 9 FU Zuodi, ZHAO Zihou, WANG Lianying. Study on the relationship between hyperuricemia and obesity in Beijing community population [J]. Chinese Journal of Diabetes, 2021, 29(1):30-34. DOI:10.3969/j.issn.1006-6187.2021.01.007.

10. Honghong $\mathrm{Lu}$, Zhirong Guo, Shijun Liu, et al. Relationship of serum uric acid concentration with metabolic syndrome and its components[J]. Chinese Journal of Diabetes, 2008, 16(5):274-277. doi: 10.3321/j.issn:1006-6187.2008.05.007.

11. Xinyu Zhou, Yiyun Liu, Yinxing Ni, et al. Relationship between hyperuricemia and abdominal obesity [J]. International Journal of Endocrinology and Metabolism, 2010, 30(z1):61-63. DOI:10.3760/cma.j.issn.1673-4157.2010.s1.022. 